\title{
POINT-CONTACT EXPERIMENTS IN URANIUM-PLATINUM COMPOUNDS
}

\author{
A G M JANSEN ${ }^{+}$, A de VISSER*, A M DUIF ${ }^{+}$, J J M FRANSE* \\ and $\mathbf{J}$ A A J PERENBOOM ${ }^{\times}$ \\ ${ }^{+}$Max-Planck-Insttut fur Festkorperforschung Hochfeld-Magnetlabor 25, Avenue des Martyrs, 166X, F-38042 Grenoble \\ Cedex, France \\ * Natuurkundig Laboratorum der Universitett van Amsterdam, Valckenterstraat 65, 1018 XE Amsterdam, The Netherlands \\ ${ }^{\times}$Research Institute for Materials, University of Nijmegen, Toernooiveld, 6525 Nijmegen, The Netherlands
}

\begin{abstract}
The resistance of metallic constrictions with $\mathrm{UPt}, \mathrm{UPt}_{2}, \mathrm{UPt}_{3}$ and $\mathrm{UPt}_{5}$ has been measured as a function of the applied voltage at low temperatures Using a heatıng model, these curves have been quantitatively compared with bulk resistivity data
\end{abstract}

Small constrictions between metals have been used to analyse the interaction of the conduction electrons with elementary excitations in a metal By measuring the resistance of a point contact as a function of the applied voltage, direct information is obtained about the energy dependent scattering of the conduction electrons Important criterion for the applicability of this method is, that the inelastic mean free path $l_{1}$ of the electrons is larger than the contact diameter $d$ In this ballistic regime the electronic system will be out of equilibrium with respect to the elementary excitations ( $1 \mathrm{e}$ phonons) in the presence of an applied voltage over the contact Mainly, the point-contact method has been applied to the study of the electron-phonon interaction $[1,2]$, although, other excitations (paramagnetic impurities [3], magnons [4], crystalfield levels [5]) have been probed with this technique In the impure limit of a contact $\left(l_{1}<\right.$ d) the electrons stay in equilibrium with the lattice at an applied voltage and in this thermal regime heating occurs in the contact region In point contacts with ferromagnetic materials it could be shown that the Curie temperature was reached in the contact region by observing an anomaly in the differential contact resistance at a certain voltage over the contact [6]

Several point-contact experiments have been done with new materials, belonging to the class of intermediate valence compounds [7] and heavy fermion compounds [8] The non-linear current-voltage characteristics of point contacts on these compounds have been explained by the energy-dependent interconfigurational scattering of the electrons [7] and by the energydependent density of states of the electrons [8] Recently, the importance of thermal effects have been noticed for these systems $[9,10]$ We will present point-contact experiments on a set of UPt compounds The data will be analysed in terms of a heating model, pointing out the resemblance between the voltage dependence of the contact resistance and the temperature dependence of the resistivity of the bulk material

In the dirty limit $(l<d)$ the resistance of a circular contact between two semi-infinite metals is given by

$R=\rho / d$,

where $\rho$ is the resistivity of the metal In this thermal limit, the effect of Joule heatıng in the contact area has to be considered Assuming equal flow lines for the electrical and thermal conduction near the contact, one obtains for an arbitrary contact geometry at an applied voltage $V$ the relation [11]

$T_{\max }^{2}-T_{0}^{2}=V^{2} / 4 L$

for the maximum temperature $T_{\max }$ at the center of the contact in a bath at temperature $T_{0}$ In this expression, $L$ is the Lorenz number which relates the electrical resistivity $\rho$ and the thermal conductivity $\lambda$ in the Wiedemann-Franz law 
$\rho \lambda=L T$ For $T_{\max } \gg T_{0}$, a numerical calculation gives $T_{\text {max }}=32 \mathrm{~V}$ with the temperature $T_{\max }$ in $\mathrm{K}$ and the voltage $V$ in $\mathrm{mV}$ Hence, using eqs (1) and (2), a simple relation exists between the voltage dependent contact resistance and the temperature dependent resistivity, if the resistance of the constriction is mostly determined by the resistivity at the center of the contact where the temperature is maximal Using the model of spherical spreading out for a constriction [11], the temperature gradient near the contact can be taken into account for the contact resistance

$R=(1 / d) \int_{0}^{1} \rho\left(T_{\max } \sqrt{1-x^{2}}\right) \mathrm{d} x$

The current-voltage characteristics were measured for point contacts of polycrystalline $\mathrm{UPt}, \mathrm{UPt}_{2}, \mathrm{UPt}_{3}$ and $\mathrm{UPt}_{5}$ At hquid $\mathrm{He}$ temperatures the contacts between the UPt compounds were adjusted by pressing the sharp edges of two pieces of bulk material against each other Prior to mounting, the bulk material was cleaved at room temperature The contact resistances were of the order of $1 \Omega$ Using eq (1) with the values for the bulk resistivity at low temperatures [12], we get diameters $d \geqslant 10 \mathrm{~nm}$ for the investigated materials, the mean free path of the electrons in these compounds will be smaller For each compound we measured the differential resistance $\mathrm{d} V / \mathrm{d} I$ of a contact as a function of the voltage For these contacts between the same materials the $\mathrm{d} V / \mathrm{d} I$ characteristics were symmetric with respect to the applied voltage Because the static contact resistance is given in eq (3), we calculated the static resistance from the $\mathrm{d} V / \mathrm{d} I$ curves In fig 1 we have plotted the relative change $\Delta R / R(V=0)$ in the static resistance of point contacts with the UPt compounds We looked at the width in the minımum of the measured resistance curves to estımate the reproducibility of the curves for different contacts For the case of $\mathrm{UPt}_{3}$ and $\mathrm{UPt}_{5}$ the width was equal within roughly $10 \%$ For UPt and $\mathrm{UPt}_{2}$ the width varied up to $30 \%$

A strong resemblance is seen between the voltage dependence in the contact resistance (fig 1) and the temperature dependence in the

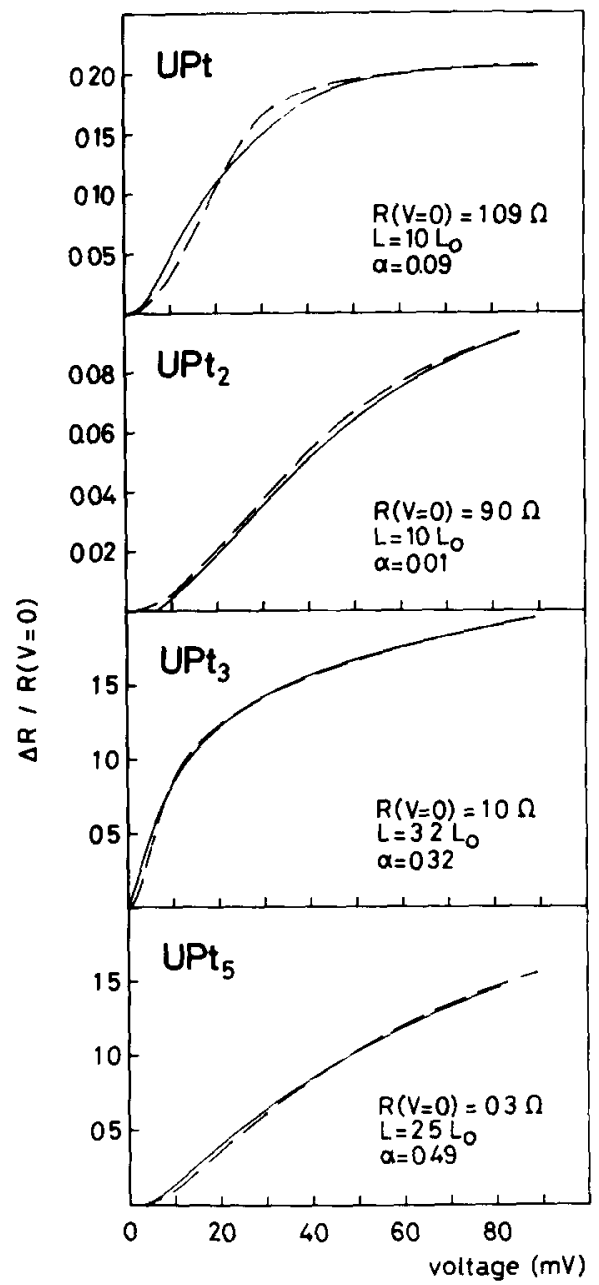

Fig 1 Normalized change in the statıc reststance as a function of the applied voltage for point contacts with uraniumplatınum compounds at liquid helium temperatures The dashed curves have been calculated with the heating model, using temperature dependent resistivity data with the effective Lorenz ratios $L$ (Sommerfeld value $L_{0}=245 \times$ $\left.10^{-8} \mathrm{~V}^{2} / \mathrm{K}^{2}\right)$, and multiplied with the factor $\alpha$

bulk resistivity [12] The observed S-shape in the point-contact data and the resistivity data is ascribed to the scattering of the electrons by spin fluctuations of the f-electrons in the case of $\mathrm{UPt}_{2}, \mathrm{UPt}_{3}$ and $\mathrm{UPt}_{5}$, and to the scattering with magnons in the case of UPt (magnetic ordering below $27 \mathrm{~K}$ )

In order to make a quantitative comparison for the heatıng model, we used eqs (2) and (3) to calculate the point-contact resistance as a 
function of the applied voltage For the conversion of the temperature scale to the voltage scale we took in eq (2) a constant Lorenz number over the whole temperature interval The results of these calculations with the used effective Lorenz numbers have been plotted in fig 1 as dashed curves To get a good agreement of the calculated curves with the experımental ones, we multiplied the calculated values of $\Delta R / R(V=0)$ by a constant $\alpha$, smaller than 1 An explanation for this constant $\alpha$ could be that part of the contact resistance is a Sharvin-like resistance $R_{0} \sim \rho l / d^{2}$, which applies for a clean contact $(l>d)$ and does not depend on the temperature The constant $\alpha$ is different for different contacts of one material, because of the difference in relative change of contact resistance For UPt and $\mathrm{UPt}_{2}$ we mostly find small values for $\alpha$, meanıng that the Sharvin resistance is much larger than the temperature dependent resistance in eq (1) In this way we find a discrepancy, because no longer the limit $l<d$ will hold and the heating model does not apply to our problem Note however, the resistivity at the constriction is different from the bulk resistivity, due to anisotropy, boundaries, strain, impurities etc Therefore, to give a better quantitative comparison, it is necessary to measure the temperature dependence of the resistivity in the point-contact area For $\mathrm{UPt}_{3}$ we have taken in fig 1 the Lorenz ratio as obtained from measurements on the electrical and thermal conductivity [13], which yielded a constant value above $70 \mathrm{~K}$ For the other curves we have taken the Lorenz number, that optımızes the agreement between experiment and theory The accuracy of the given Lorenz numbers must be seen in view of the, before mentioned, reproducibility of the width in the minimum of the resistance curves A Lorenz number much larger than the Sommerfeld value $L_{0}=$ $245 \times 10^{-8} \mathrm{~V}^{2} / \mathrm{K}^{2}$ would mean that the phonon part in the thermal conductivity plays an important role compared to the electronic part [13]

In the investigated UPt compounds a resemblance exists between the point-contact resistance as a function of the applied voltage and the bulk resistivity as a function of the temperature However, to give a detalled description of this heatıng model, the measured curves could only be reproduced by adding a constant resistance (independent of temperature) to the calculated contact resistance Especially for UPt and $\mathrm{UPt}_{2}$, ascribing this constant resistance to an appreciable Sharvin-like resistance would be in contradiction with the condition $l<d$, which is necessary for the applicability of the heating model Recently, point-contact data on $\mathrm{UPt}_{3}$ were analysed in a measurement of the energy dependent density of states of the electrons [8] In detall it is not yet clear how the energy dependent mass-renormalization enters the point-contact problem We believe that hedting plays a role in the description of point-contact experıments with these metallic compounds

\section{References}

[1] A G M Jansen, A P van Gelder and P Wyder, J Phys C13 (1980) 6073

[2] I K Yanson, Sov J Low Temp Phys 9 (1983) 343 [Fiz Nizk Temp 9 (1983) 676]

[3] A G M Jansen, A P van Gelder, P Wyder and S Strassler, J Phys F 11 (1981) L15

[4] A I Akımenko and I K Yanson, JETP Lett 31 (1980) 191 [Pis'ma Zh Eksp Teor Fiz 31 (1980) 209]

[5] A I Akımenko, NM Ponomarenko, I K Yanson, S Janoš and M Reiffers, in Proc LT-17, eds U Eckern, A Schmid, W Weber and $\mathrm{H}$ Wuhl (North-Holland, Amsterdam, 1984) p 1085

[6] B I Verkın, I K Yanson, I O Kulık, O I Shklyarevskı, A A Lysykh and Yu G Naydyuk, Solid State Commun 30 (1979) 215

[7] B Bussian, I Frankowskı and D Wohlleben, Phys Rev Lett 49 (1982) 1026

[8] M Moser, P Wachter, J J M Franse, G P Meisner and E Walker, J Magn Magn Mat 54-57 (1986) 373

[9] E Paulus and G Voss, J Magn Magn Mat 47\&48 (1985) 539

[10] Yu G Neĭdyuk, N N Grivbov, A A Lysykh, I K Yanson, N B Brandt and V V Moshchalkov, JETP Lett 41 (1985) 399 [Pis'ma Zh Eksp Teor Fiz 41 (1985) 325]

[11] R Holm, Electric Contacts (Springer Verlag, Berlın 1967)

[12] A de Visser, J J M Franse and A Menovsky, J Magn Magn Mat 43 (1984) 43

[13] J J M Franse, A Menovsky, A de Visser, C D Bredl, U Gottwick, W Lieke, H M Mayer, U Rauchschwalbe, G Sparn and F Steglich, Z Phys B59 (1985) 15 